\title{
METODOLOGÍA E IMPLEMENTACIÓN DE SIX SIGMA
}

\section{METHODOLOGY AND IMPLEMENTATION OF SIX SIGMA}

\author{
Eduardo Navarro Albert ${ }^{1}$ \\ Víctor Gisbert Soler ${ }^{2}$ \\ Ana Isabel Pérez Molina ${ }^{3}$
}

1. Graduado en Ingeniería Electrónica y Automática Industrial. Máster en Ingeniería en Organización y Logística. Universidad Politécnica de Valencia, (España). E-mail: ednaal@epsa.upv.es

2. Doctor Ingeniero Industrial. Departamento de Estadística e Investigación Operativa Aplicadas y Calidad. Universidad Politécnica de Valencia, (España). E-mail: vgisber@eio.upv.es

3. Ingeniera Técnico Industrial Química. Ingeniera en Organización Industrial. Doctor por la Universidad Politécnica de Valencia. Departamento de Estadística, (España). E-mail: anpemo@eio.upv.es

Citación sugerida:

Navarro Albert, E., Gisbert Soler, V. y Pérez Molina, A.I. (2017). Metodología e implementación de Six Sigma. 3C Empresa: investigación y pensamiento crítico, Edición Especial, 73-80. DOI: <http://dx.doi.org/10.17993/3cemp.2017.especial.73-80/>. 


\section{RESUMEN}

Este artículo presenta la metodología e implementación del modelo Six Sigma en los diferentes tipos de organizaciones. Six Sigma es una herramienta con la finalidad de medir y mejorar la calidad. Se define como una metodología basada en datos para conseguir la calidad más cercana a la perfección. Esto se consigue examinando los procesos productivos de manera exhaustiva.

Seis Sigma es diseño, comunicación, formación, producción, administración, etc. Para la implantación de esta metodología se requieren principalmente dos cosas: tiempo y compromiso. También requiere de una inversión económica que en poco tiempo se convertirá en un ahorro en costes para la empresa y en una mejora para los procesos internos de la organización.

\section{ABSTRACT}

This article presents the methodology and implementation of the Six Sigma model in different types of organizations. Six Sigma is a tool with the purpose of measuring and improving quality. It is defined as a data-based methodology to achieve the closest quality to perfection. This is achieved by examining the production processes in a comprehensive manner.

Six Sigma is design, communication, training, production, administration, etc. For the implementation of this methodology, two things are mainly required: time and commitment. It also requires an economic investment that in a short time will become a cost savings for the company and an improvement for the internal processes of the organization.

\section{PALABRAS CLAVE}

Seis Sigma, DMAIC, Calidad, Mejora continua, Cinco ¿Por qué?

\section{KEY WORDS}

Six Sigma, DMAIC, Quality, Continuous Improvement, Five Why? 


\section{INTRODUCCIÓN}

El método Seis Sigma es una filosofía que apareció en los años ochenta gracias al ingeniero Mikel Harry, a través de la evaluación y análisis de la variación de los procesos en la empresa Motorola. Fue la primera empresa en implantar esta metodología como estrategia de mercado y de mejoramiento de la calidad. Debido a la globalización, las empresas del sector industrial y comercial empezaron a desarrollar técnicas para optimizar los procesos y mejorar su competitividad y productividad. Esta metodología también se enfoca en la mejora continua.

Basada en los conceptos estadísticos de Shewart, Deming, Juran y Taguchi, seis sigma aporta soluciones a corto plazo de problemas repetitivos. Se compone de un diseño robusto además de establecer tolerancias para definir un estándar y saber que productos tienen o no la suficiente calidad para salir al mercado.

\section{ANTECEDENTES}

Los artículos que se han encontrado como antecedentes relacionados con el modelo Six Sigma son los siguientes:

En el artículo "Plan de implementación Six Sigma en el proceso de admisión de una institución de educación superior" [1]. Se siguieron los siguientes pasos para poner este plan en marcha:

- En primer lugar, se realizó un DAFO para detectar los puntos fuertes y débiles tanto internos como externos del proceso.

- Se elaboró un diagrama causa-efecto.

- Se desarrolló los cinco ¿por qué? En el proceso de admisiones.

Este artículo concluye que gracias a esta metodología es posible clasificar las causas principales de los errores de un proceso para encontrar las mejores soluciones y evitar su reaparición. Después de haber implementado esta metodología en un IES, recomiendan antes de nada se necesita la sensibilización de todos los asociados al Institución y el compromiso de la misma, disciplina, medición y documentación de la información.

- El artículo "Aplicación de Seis Sigma en una microempresa del Ramo Automotriz" [2], basa su éxito en los siguientes puntos:

- La necesidad de la organización por mejorar.

- El fuerte apoyo por parte de la gerente general de la empresa.

- Cada integrante asumió su rol y el liderazgo correspondiente para solucionar los problemas trabajando equipo.

- Se eliminaron las barreras entre los departamentos a través de constantes reuniones.

- Cuando las mejoras implementadas no generaban el resultado de la empresa, el apoyo del líder de la empresa era indispensable para afrontar este tipo de situaciones. 
- Aplicación de la metodología DMAIC.

$>$ En el artículo "Aplicación de la metodología Seis Sigma para reducir la pérdida de café al granel en una planta de envasado" [3]. La efectividad de la utilización de la metodología DMAIC superó los resultados esperados. Los factores de éxito de este proyecto fueron los siguientes:

- El soporte del gerente y su involucramiento constante durante todo el proyecto.

- La experiencia y el conocimiento del proceso por parte del desarrollador del proyecto.

- El análisis de causas potenciales.

- El diseño del experimento.

> En el artículo "Aplicación de la metodología Seis Sigma en la mejora de resultados de los proyectos de construcción" [4], se concluyó lo siguiente:

- La falta de calidad en las construcciones provoca costes económicos y sociales muy elevados.

- La filosofía Seis Sigma es aplicable en este campo debido a que todos los proyectos de construcción comparten fases comunes pudiendo hacer uso de las herramientas Seis Sigma.

- El cambio de actitud del redactor de proyecto fue indispensable para el éxito de la aplicación de esta metodología.

\section{METODOLOGÍA SEIS SIGMA}

Seis Sigma es una metodología compuesta por cinco fases: Definir, Medir, Analizar, Mejorar y Controlar. Representa el número el número de desviaciones estándar obtenidas a la salida del proceso. Su objetivo de aumentar la capacidad de los procesos, de tal forma que estos generen los mínimos defectos por millón de unidades producidas. Estos defectos deben ser imperceptibles para el cliente.

Actualmente muchas de las organizaciones cuentan con este sistema como estrategia de negocio para aumentar su rentabilidad y mejorar la calidad de sus productos y servicios.

Seis Sigma es una metodología basada en cinco principios:

- Enfoque al cliente.

- Centrado en los procesos.

- Metodología para la realización de proyectos.

- Estructura organizacional.

- Lucha contra la variación.

La calidad es uno de los factores principales para la satisfacción de los clientes, y el medio para lograr atraerlos y mantenerlos. Para lograr la calidad es imprescindible implantar en 
los miembros y en la cultura de la organización una actitud siempre desde la perspectiva del cliente.

Esta herramienta se centra en la mejora de los procesos enfocándose en los aspectos críticos para el cliente. Mediante la medición de los diferentes procesos reduciendo el número de defectos para que la producción pueda continuar de forma común.

Dicha metodología propone dos campos de aplicación: implementación de un proyecto existente o la creación de un nuevo proyecto, producto o servicio. Estos campos se centran en la reducción de defectos, fallos y no conformidades tratando de conseguir un valor cercano a 0.

El equipo de un proyecto Seis Sigma consta de los siguientes tipos de miembros:

- Champion: es un miembro de la dirección. Se encarga de evitar conflictos de interés entre departamentos además de participar en la elección de proyectos y es informado del avance de los proyectos.

- Black Belt: experto en la metodología seis sigma. Se encarga de los proyectos que necesitan el uso de herramientas de calidad y técnicas estadísticas avanzadas.

- Green Belt: se encarga de llevar los proyectos seis sigma, de informar al champions, forma a los miembros del equipo sobre las técnicas seis sigma y asegura el mantenimiento de los logros obtenidos en el proyecto.

- Coordinador Lean 6 Sigma: se encarga de seguir todos los proyectos, manteniendo reuniones regulares con los Green Belt y/o Black Belt e informando a los diferentes champions del avance global de la iniciativa.

En un principio este equipo se componía de los diferentes roles mencionados anteriormente, pero al poner en la práctica esta metodología se añadió un nuevo rol. Este nuevo rol se denomina Yellow Belts, participan en los proyectos Seis Sigma ayudando a los Green Belts y Black Belts.

El método Seis Sigma conocido también como DMAIC (Definir, Medir, Analizar, Mejorar y Controlar) se estructura en cinco fases.

- Fase de definición: se identifican los proyectos Seis Sigma que deben ser evaluados por la dirección para evitar la infrautilización de recursos, para así asignar la prioridad necesaria para cada proyecto.

- Fase de medición: consiste en la caracterización del proceso identificando los requisitos clave de los clientes, las características clave del producto y los parámetros que afectan al funcionamiento del proceso y a las características clave. Es donde se define el sistema de medida y se mide la capacidad del proceso.

- Fase de análisis: se analizan los datos actuales e históricos. Se desarrollan hipótesis sobre posibles relaciones causa-efecto mediante el uso de herramientas estadísticas. 
- Fase de mejora: se determina la relación causa-efecto para predecir, mejorar y optimizar el funcionamiento del proceso.

- Fase de control: se diseñan y documentan los controles necesarios para asegurar que el sistema implantado se mantenga en el tiempo.

\section{IMPLEMENTACIÓN SEIS SIGMA}

La letra sigma es utilizada en estadística para el cálculo de la desviación estándar de la muestra en la siguiente ecuación:

$S=\sqrt{\frac{\sum i=1\left(X_{1}-X\right)^{2}}{(n-1)}}$

Donde las variables son:

s= Desviación estándar de la muestra

$\mathrm{X} i=$ datos de la muestra para $\mathrm{i}=1,2,3$.

$\mathrm{X}=$ Promedio de la muestra (media)

$\mathrm{n}=$ Número de datos de la muestra

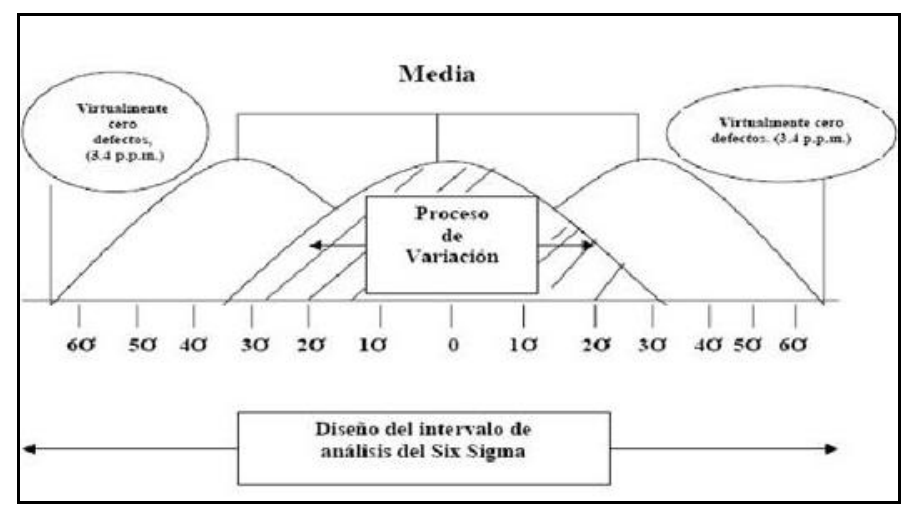

Ilustración 1. Representación gráfica six sigma.

El objetivo de esta metodología es obtener 3, 4 defectos por millón de oportunidades. Clasificando la eficiencia de un proceso con base a su nivel sigma:

- 1 sigma $=68,27 \%$ de eficiencia.

- 2 sigma $=95,45 \%$ de eficiencia.

- 3 sigma $=99,73 \%$ de eficiencia.

- 4 sigma $=99,994 \%$ de eficiencia.

- 5 sigma $=99,99994 \%$ de eficiencia.

- 6 sigma $=99,9999966 \%$ de eficiencia. 


\section{CONCLUSIONES}

En la actualidad estamos delante de un mercado que con los avances tecnológicos cambia de manera constante. El objetivo de las organizaciones es diferenciar sus productos o servicios potenciándolos en diversas características ya sea en liderazgo en precios o mejorando la calidad.

Una opción óptima para realizar un análisis y conocer que características podríamos explotar en un producto sería con el uso de la metodología Seis Sigma. A través del método DMAIC podremos conseguir un uso optimizado de los recursos, reducción de costes... para así aumentar la competitividad frente a las empresas competidoras. También nos puede facilitar una definición más clara de la visión de la empresa al realizar un amplio análisis de todos los procesos.

Como conclusión de este artículo, cabe destacar que en todos los antecedentes de esta metodología e implementación coinciden en que el apoyo absoluto del responsable de proyecto, la implicación de los equipos de trabajo asumiendo sus diferentes roles y la necesidad de mejora de las organizaciones son los factores potenciales para esta aplicación. 


\section{REFERENCIAS BIBLIOGRÁFICAS}

[1] Arango Martínez D.M., Ángel Álvarez B.E. "Plan de implementación Six Sigma en el proceso de admisión de una institución de educación superior". Prospect. Vol. 10, No. 2 , Julio - Diciembre de 2012, págs. 13-21.

[2] Flores Ávila E., Varela Loyola J.A., Gallardo García D., Tolamatl Michcol J. “Aplicación de Seis Sigma en una microempresa del Ramo Automotriz". Conciencia Tecnológica No. 42, Julio-Diciembre 2011.

[3] Buestán M. "Aplicación de la metodología Seis Sigma para reducir la pérdida de café al granel en una planta de envasado". LACCEI August 14-16 Cancún, México.

[4] Yepes Víctor E., "Aplicación de la metodología Seis Sigma en la mejora de resultados de los proyectos de construcción". UPV 